\title{
Dislipidemia em crianças e adolescentes do município de Marau-RS
}

\author{
Dyslipidemia in children and adolescents of the \\ municipality of Marau-RS
}

\author{
Suellen Savi Calliari ${ }^{1}$ (D), Luciana Grazziotin Grando ${ }^{1}$ (D), Charise Dellazen Bertol ${ }^{1}$ (D), \\ Luciano de Oliveira Siqueira $^{1}$ (iD
}

\begin{abstract}
Resumo
Introdução: As doenças cardiovasculares são consideradas um dos principais problemas de saúde na atualidade. O perfil lipídico de crianças e adolescentes se associa diretamente com a prevalência de doença coronariana em adultos, pois o processo aterosclerótico começa a se desenvolver na juventude e progride na vida adulta. Objetivo: Avaliar o perfil lipídico de crianças e adolescentes da cidade de Marau-RS. Método: Realizou-se um estudo retrospectivo, no qual foi avaliado o perfil lipídico de crianças e adolescentes de 1 a 19 anos, período de abril de 2016 a julho 2017, totalizando 272 pacientes. Resultados: Entre os pacientes analisados, 34,7\% apresentaram hipercolesterolemia, 56,7\% demonstraram níveis de HDL abaixo do desejável, 16\% exibiram níveis de LDL aumentado e 16\% tinham hipertrigliceridemia. A análise estatística dos resultados mostrou uma concentração de colesterol total, HDL e triglicerídeos significativamente mais alta em meninas do que em meninos. Conclusão: $\mathrm{O}$ percentual elevado de dislipidemias na infância aponta para a necessidade de implantação de políticas públicas que previnam a dislipidemia juvenil, minimizando, assim, agravos relacionados a doenças cardiovasculares na vida adulta da população.
\end{abstract}

Palavras-chave: crianças; adolescentes; perfil lipídico; dislipidemias; doenças cardiovasculares.

\begin{abstract}
Background: Cardiovascular diseases are considered one of the main health conditions nowadays. The lipid profile of children and adolescents is directly associated with the prevalence of coronary disease in adults, since the atherosclerotic process begins to develop in youth and progresses in adult life. Objective: To evaluate the lipid profile of children and adolescents in the city of Marau-RS. Method: A retrospective study was carried out to evaluate the lipid profile of children and adolescents aged 1 to 19 years, from April 2016 to July 2017, a total of 272 patients. Results: Among the patients analyzed, 34.7\% presented hypercholesterolemia, $56.7 \%$ had below-desirable HDL levels, $16 \%$ had increased LDL levels and $16 \%$ had hypertriglyceridemia. Statistical analysis of the results shows a significantly higher concentration of total cholesterol, HDL and Triglycerides in girls when compared to boys. Conclusion: The high percentage of dyslipidemia in childhood points to the need to implement public policies for the prevention of juvenile dyslipidemia. Thus minimizing cardiovascular diseases related to adult life in the population.
\end{abstract}

Keywords: children; teens; lipid profile; dyslipidemia; cardiovascular diseases.

${ }^{1}$ Curso de Farmácia, Instituto de Ciencias Biológicas, Universidade de Passo Fundo (UPF) - Passo Fundo (RS), Brasil.

Trabalho realizado no município de Marau (RS), Brasil.

Endereço para correspondência: Luciano de Oliveira Siqueira, BR 285, Km 171 - Bairro São José - CEP: $99052-900$ - Passo Fundo (RS), Brasil -

Email: luciano@upf.br

Fonte de financiamento: nenhuma.

Conflito de interesses: nada a declarar. 


\section{INTRODUÇÃO}

As doenças cardiovasculares (DCV) constituem um dos principais problemas de saúde pública dos tempos atuais, diminuindo significativamente a vida produtiva e levando à mortalidade precoce ${ }^{1}$. Níveis elevados de colesterol total, lipoproteína de baixa densidade (colesterol LDL) e triglicerídeos estão relacionados com o maior risco de doença aterosclerótica ${ }^{2-5}$

A aterogênese é um processo que tem início na infância de maneira silenciosa e progride a partir da terceira década de vida $^{6}$. Estrias gordurosas, precursoras das placas ateroscleróticas, começam a aparecer na camada íntima da aorta aos 3 anos de idade ${ }^{7}$ e nas coronárias durante a adolescência, podendo progredir significativamente na terceira e quarta décadas de vida. O desenvolvimento da doença aterosclerótica (DAC) sintomática tem sido correlacionado a fatores de risco para a aterosclerose ${ }^{8}$. Entre os principais fatores estão histórico familiar de DAC, dislipidemia, hipertensão arterial, diabetes melito, obesidade, tabagismo e sedentarismo ${ }^{9,10}$.

A dislipidemia, condição em que há concentrações anormais de lipídeos ou lipoproteínas no sangue, é um fator de risco importante para agravo e complicações tardias da aterosclerose. Há uma forte correlação entre o risco para DAC e concentrações séricas elevadas de colesterol total (CT), particularmente de lipoproteínas de baixa densidade (LDL), assim como concentrações reduzidas de lipoproteínas de alta densidade (HDL) ${ }^{11,12}$.

As dislipidemias não são exclusivas de adultos. Hábitos relacionados ao consumo exagerado de alimentos industrializados/processados e sedentarismo têm aumentado a prevalência de dislipidemias em crianças e adolescentes. Estima-se que $38,5 \%$ das crianças no mundo apresentem dislipidemia em diferentes graus. A herança genética, o gênero, a idade e os hábitos de vida têm grande importância para o desenvolvimento de um perfil lipídico inadequado ${ }^{1}$. A associação de dislipidemia com obesidade pode resultar em resistência insulínica, provocando a síndrome metabólica, cuja prevalência em crianças e adolescentes vem aumentando por causa do aumento das taxas de sobrepeso e obesidade em crianças entre 2 e 5 anos $^{13}$.

Os níveis elevados de colesterol em crianças se associam diretamente à prevalência de doença coronariana nos adultos ${ }^{14,15}$. Isso pode ser explicado pelo fenômeno de trilha (tracking), em que há uma forte tendência de crianças manterem os mesmos percentis de colesterol até a vida adulta ${ }^{16,17}$. Os fatores de risco para doença aterosclerótica tendem a se agrupar nos indivíduos (clustering effect), mantendo-se no processo de crescimento e desenvolvimento ${ }^{18}$.

Estudos epidemiológicos realizados no Brasil apresentam diferentes graus de incidência de dislipidemia na infância e na juventude. Etnias, miscigenação e aspectos genotípicos e fenotípicos de um país de extensão continental justificariam a divergência desses dados.
A cidade de Marau, que se localiza no planalto médio do Rio Grande do Sul, é um município de 40 mil habitantes colonizado por imigrantes italianos em 1904. Aspectos genotípicos combinados a hábitos alimentares típicos da descendência italiana (consumo elevado de embutidos, laticínios, compotas de frutas, geleias caseiras, produtos coloniais e panificados caseiros) podem influenciar a prevalência de casos de dislipidemia nessa população de crianças e adolescentes e, consequentemente, modificar o risco de doenças cardiovasculares na população adulta em um futuro próximo.

Partindo desses princípios, avaliou-se o perfil lipídico de crianças e adolescentes da cidade de Marau-RS como forma de estimar o risco de doença cardiovascular na região e contribuir para políticas públicas de intervenção.

\section{MÉTODO}

\section{Delineamento}

O presente estudo é retrospectivo, no qual foi avaliado o perfil lipídico de jovens que realizaram exames de colesterol total (CT), triglicerídeos (TG), HDL e LDL na cidade de Marau-RS.

\section{Casuística}

Foi utilizada como fonte de cálculo amostral a população residente na cidade de Marau (população de 36.364 habitantes, segundo o censo do IBGE de 2010) ${ }^{19}$. Assumindo estimativa de $40 \%$ de a população residente ter menos de 19 anos, determinou-se a necessidade da participação de 272 indivíduos para a realização de um estudo com margem de erro de 3\% e intervalo de confiança de 95\%. Assim, foram incluídos na amostra 272 indivíduos de ambos os sexos, de 1 a 19 anos de idade, que realizaram exames laboratoriais na cidade de Marau-RS no período de abril de 2016 a julho 2017. Os dados foram obtidos a partir de resultados de exames laboratoriais obtidos no sistema de informática JALIS do laboratório. Previamente à realização dos exames, procedeu-se a uma anamnese padrão com relação ao consumo de alimentos, ao uso de medicamentos e ao jejum. Foram incluídos no estudo todos os pacientes que fizeram os exames com 12 horas de jejum, que não faziam uso de medicamentos, com dieta estável de 72 horas e que não tinham histórico familiar de dislipidemia e doença cardiovascular. Todos os indivíduos eram estudantes da rede pública e privada. Foram excluídos aqueles que não estavam em jejum ou que faziam uso de algum tipo de medicamento no momento da realização do exame.

\section{Protocolo experimental}

Procedeu-se assepticamente à coleta de amostras de $8 \mathrm{~mL}$ de sangue venoso mediante punção na fossa antecubital. As amostras de sangue foram incubadas a $37^{\circ} \mathrm{C}$ para coagulação e retração do coágulo. A seguir, o sangue foi centrifugado a $2.000 \mathrm{rpm}$ 
por 10 minutos. O soro foi extraído e acondicionado em frascos Eppendorf para posterior análise bioquímica, a qual foi constituída da determinação de triglicerídeos (glicerol fosfato oxidase/peroxidase - Biosystems ${ }^{\circledR}$ ), colesterol total (método colesterol oxidase/peroxidase - Biosystems ${ }^{\circledR}$ ) e colesterol HDL (método de precipitação - Biotecnica ${ }^{\circledR}$ ), seguindo as recomendações do fabricante. Após a execução técnica, as concentrações dos analitos foram determinadas em analisador bioquímico COBAS MIRA PLUS - Roche ${ }^{\circledR}$. Para as frações de VLDL e LDL, aplicou-se a equação de Friedwald.

\section{Aspectos éticos}

O trabalho foi desenvolvido segundo declarações e diretrizes sobre pesquisas que envolvem seres humanos: Código de Nuremberg, Declaração de Helsinque e Resolução nº 196, de 10 de outubro de 1996, do Conselho Nacional de Saúde. Também foi aprovado e regulamentado pelo Comitê de Ética da instituição executora, sob Parecer no 804.641/2014.

\section{Análise dos resultados}

Os resultados foram transcritos em uma planilha de trabalho para análise estatística descritiva e inferencial. A normalidade da amostra foi testada mediante o teste Kolmogorov-Smirnov. Para resultados com distribuição paramétrica, foram aplicados os testes T de Student, empregando-se nível de significância de $\mathrm{p}<0,05$.

\section{RESULTADOS}

Neste estudo, foram avaliadas 272 pessoas, das quais 145 (53,3\%) do gênero feminino, e 127 (46,7\%), do gênero masculino. A média de idade dos participantes foi de 13,8 anos para as meninas e 13,2 anos para os meninos.

A análise dos resultados mostra uma diferença estatisticamente significante entre os sexos, em que as meninas apresentaram uma concentração de CT, HDL, VLDL e TG maior que os meninos (Tabela 1).

Para análise do risco de doença cardiovascular com base no perfil lipídico, adotaram-se os valores de referência preconizados na Atualização da Diretriz Brasileira de Dislipidemias e Prevenção da Aterosclerose ${ }^{1}$ (2017). Dos 259 pacientes que fizeram dosagem de CT, 90 (64 do sexo feminino e 26 do sexo masculino) estavam com concentração elevada (CT $>170 \mathrm{mg} / \mathrm{dL}$ ), 50 (28 do sexo feminino e 22 do sexo masculino), com a dosagem limítrofe (CT 151-170 mg/dL), e 119 (45 do sexo feminino e 74 do sexo masculino), com CT desejável (CT $<150 \mathrm{mg} / \mathrm{dL}$ ) (Figura 1). A análise estatística dos resultados entre os sexos mostrou uma elevação estatisticamente significante no sexo feminino $(171,1 \pm 3,1 \mathrm{mg} / \mathrm{dL})$ quando comparado com o sexo masculino $(152,4 \pm 3,2 \mathrm{mg} / \mathrm{dL})$ (Tabela 1$)$.

Quanto ao colesterol HDL, dos 201 pacientes avaliados, 87 (56 do sexo feminino e 31 do sexo masculino) apresentaram uma concentração desejável (> $45 \mathrm{mg} / \mathrm{dL}$ ) e 114 (50 do sexo feminino e 64 do sexo masculino) estavam com o valor de HDL inferior a $45 \mathrm{mg} / \mathrm{dL}$ (Figura 2). A análise estatística dos resultados entre os sexos, mostrou uma elevação estatisticamente significante no sexo feminino $(46,9 \pm 1,1 \mathrm{mg} / \mathrm{dL})$ quando comparado ao sexo masculino $(42,0 \pm 1,0 \mathrm{mg} / \mathrm{dL})$ (Tabela 1$)$.

A concentração de TG no sexo feminino $(93,5 \pm 3,7 \mathrm{mg} / \mathrm{dL})$ apresentou uma concentração estatisticamente superior ao sexo masculino (78,8 $\pm 3,6 \mathrm{mg} / \mathrm{dL}$ ) (Tabela 1). Dos 269 pacientes avaliados, 189 ( 92 do sexo feminino e 97 do sexo masculino) estavam com o valor desejável (<100 mg/dL), 35 (23 do sexo feminino

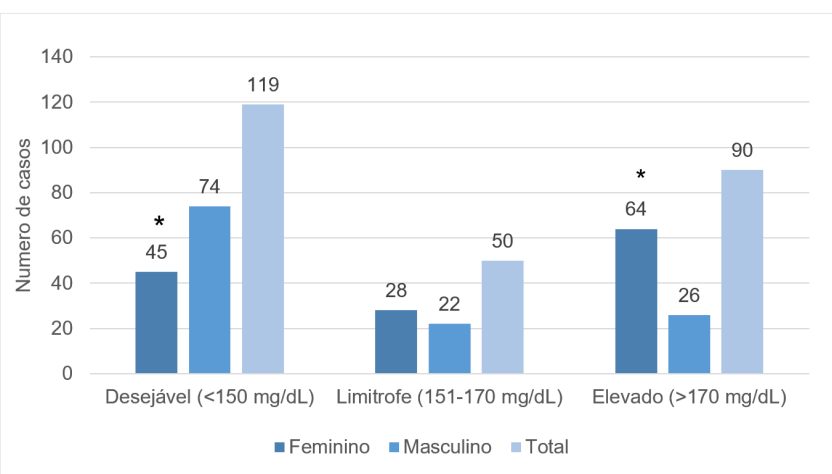

Figura 1. Estratificação da concentração de colesterol total de jovens de 1 a 19 anos de Marau-RS. Valores de corte com base na Atualização da Diretriz Brasileira de Dislipidemias e Prevenção da Aterosclerose $(2017)^{1}$. Resultados expressos em número de casos. * $p<0,05$ segundo teste T de Student para amostras independentes

Tabela 1. Valores médios de colesterol total, HDL, LDL, VLDL e triglicerídeo de jovens entre 1 e 19 anos de idade da cidade de Marau-RS

\begin{tabular}{crrr} 
& $\begin{array}{c}\text { Feminino } \\
(\mathbf{n = 1 4 5 )}\end{array}$ & $\begin{array}{c}\text { Masculino } \\
(\mathbf{n = 1 2 7 )}\end{array}$ & $\mathbf{P}$ \\
Colesterol total & $171,3 \pm 3,1$ & $152,4 \pm 3,2$ & $0,000^{\star}$ \\
Colesterol HDL & $46,9 \pm 1,1$ & $42,0 \pm 1,0$ & $0,005^{\star}$ \\
Colesterol LDL & $103,9 \pm 3,1$ & $92,8 \pm 2,7$ & 0,09 \\
Colesterol VLDL & $18,7 \pm 0,7$ & $15,7 \pm 0,7$ & $0,005^{\star}$ \\
Triglicerídeos & $93,5 \pm 3,7$ & $78,8 \pm 3,6$ & $0,005^{\star}$ \\
\hline
\end{tabular}

Valor de P obtido pelo teste T de Student para amostras independentes. Resultados expressos como média \pm desvio-padrão; ${ }^{*} \mathrm{p}<0,05$ segundo teste T de Student para amostras independentes 


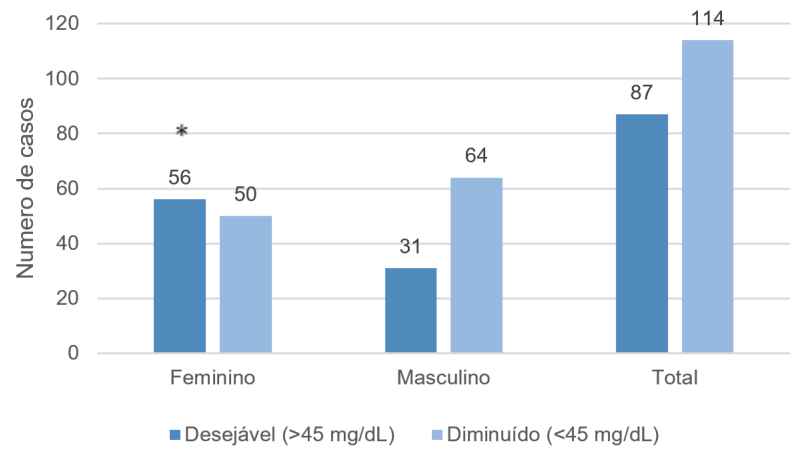

Figura 2. Estratificação da concentração de colesterol HDL de jovens de 1 a 19 anos de Marau-RS. Valores de corte com base na Atualização da Diretriz Brasileira de Dislipidemias e Prevenção da Aterosclerose $(2017)^{1}$. Resultados expressos em número de casos. ${ }^{*} p<0,05$ segundo teste T de Student para amostras independentes

e 12 do sexo masculino), na faixa limítrofe (101-130 mg/dL), e 45 ( 28 do sexo feminino e 17 do sexo masculino), com TG elevado (> $131 \mathrm{mg} / \mathrm{dL})$.

\section{DISCUSSÃO}

Estudos têm demonstrado que fatores de risco para a aterosclerose estão presentes desde a infância e persistem na vida adulta, o que torna importante a dosagem do perfil lipídico na infância como forma de prevenção de doença coronariana. A recomendação para medida do perfil lipídico se limita a crianças com histórico familiar de hipercolesterolemia ou doença coronariana precoce ${ }^{11}$. Entretanto, essa prática mostra baixa sensibilidade para detectar indivíduos com risco, conforme demonstrado em estudos internacionais e nacionais ${ }^{20,21}$. Dennison et al. ${ }^{22}$ encontraram $40 \%$ de crianças brancas e $21 \%$ de crianças negras que apresentaram hipercolesterolemia com histórico familiar de doença cardiovascular. Isso indica que, mesmo sem antecedentes na família, o fator de risco pode estar presente e, por isso, é recomendável pesquisá-lo.

O presente estudo determinou a distribuição dos lipídeos séricos de crianças e adolescentes de 1 a 19 anos. A análise dos resultados mostrou que $34,7 \%$ dos pacientes apresentavam hipercolesterolemia. O percentual de casos encontrados nesta pesquisa concorda com estudos realizados em Fortaleza ${ }^{23}(32,3 \%)$ e Campinas ${ }^{24}(44 \%)$, o que revela que a hipercolesterolemia juvenil não é um fator endêmico. Além disso, a concentração de colesterol total nas meninas $(171,3 \pm 3,1 \mathrm{mg} / \mathrm{dL})$ foi estatisticamente mais elevada quando comparada com os meninos (152,47 $\pm 3,2 \mathrm{mg} / \mathrm{dL}$ ). A diferença entre sexos pode ser justificada pelo aumento da demanda de colesterol para síntese de hormônios esteroidais envolvidos no ciclo menstrual das meninas. Por outro lado, em um estudo realizado com escolares de 7 a 18 anos no município de Florianópolis, Giuliano et al. ${ }^{2}$ indicaram que $10 \%$ dos pacientes apresentaram hipercolesterolemia.

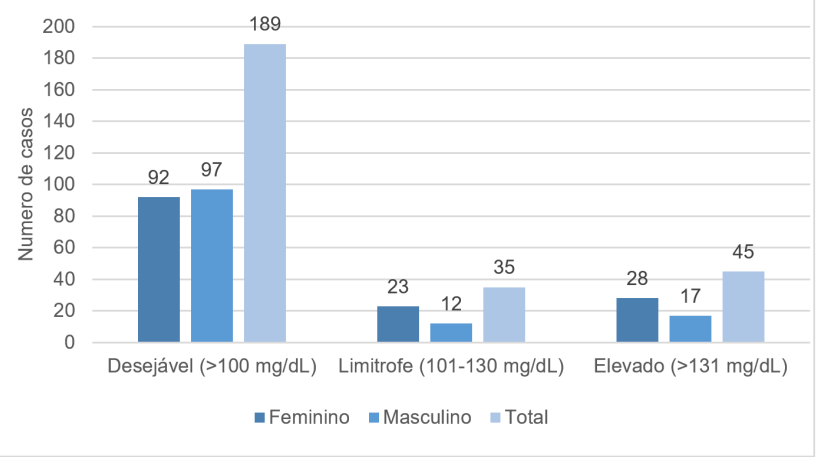

Figura 3. Estratificação da concentração de triglicerídeos de jovens de 1 a 19 anos de Marau-RS. Valores de corte com base na Atualização da Diretriz Brasileira de Dislipidemias e Prevenção da Aterosclerose (2017) ${ }^{1}$. Resultados expressos em número de casos

A análise das frações do colesterol apontou que $56,7 \%$ dos indivíduos analisados apresentaram valores de HDL abaixo do desejável para a faixa etária, dos quais os maiores níveis de HDL (ou seja, valores desejáveis) foram encontrados nos meninos $(67,3 \%)$ quando comparados com as meninas $(47,1 \%)$.

Os níveis de lipídeos e lipoproteínas sofrem variações importantes durante a fase de crescimento e desenvolvimento humano, com diferenças segundo idade, sexo e peso corporal. Os níveis séricos de lipídeos e lipoproteínas são superiores em crianças e adolescentes do sexo feminino, sendo essa diferença mais expressiva durante a adolescência. Em média, as meninas possuem níveis superiores de CT, HDL e $\mathrm{LDL}^{2-5}$. As variações decorrentes da maturação sexual ocorrem em ambos os sexos. Nas meninas, observa-se um aumento progressivo do HDL a partir dos 10 anos, o qual é marcadamente superior ao dos meninos no final da adolescência. Também o LDL e o CT se elevam progressivamente a partir dos 14-15 anos nas meninas, sendo superiores aos dos meninos por volta dos 17-18 anos ${ }^{3}$.

O LDL é a fração lipídica mais aterogênica no sangue e implica a maior prevalência de eventos cardiovasculares mórbidos na vida adulta. Os resultados apontaram que 16\% das crianças e dos adolescentes apresentaram níveis de LDL acima do recomendado. É importante destacar que existem subclasses de LDL. As partículas de LDL tipo B, pelo menor tamanho e pela maior densidade, atravessam mais facilmente a barreira endotelial e são mais facilmente oxidáveis, o que as tornam também mais aterogênicas ${ }^{25}$. Assim, mesmo em indivíduos com níveis normais de LDL, o perfil lipídico pode ser menos favorável por causa da proporção entre as subclasses das lipoproteínas ${ }^{26}$.

A Figura 3 mostra que o percentual de hipertrigliceridemia na população avaliada foi de $16,7 \%$, corroborando resultados semelhantes obtidos em estudos realizados em Belém, no Estado 
do Pará $^{27}(15,8 \%)$, e na Argentina ${ }^{28}$ (18\%), ambos realizados com jovens entre 6 e 19 anos de idade.

Atualmente, admite-se a hipótese de que algumas lipoproteínas ricas em TG podem influenciar de modo independente o desenvolvimento da aterosclerose. Estudos de necropsia mostraram uma associação de concentrações aumentadas de TG e LDL com estrias gordurosas e placas fibrosas em artérias coronárias $^{10}$.

Considerando a maior prevalência de hipercolesterolemia nas meninas em razão da menacme e do aumento da taxa de turnover do metabolismo do colesterol para síntese de hormônios esteroidais, medidas de saúde pública envolvendo ginecologistas podem contribuir para o processo de controle de dislipidemias na vida adulta.

O presente estudo limitou-se a analisar o perfil lipídico de uma população específica de uma cidade do Rio Grande do Sul como ferramenta de estudos para perfil epidemiológico com vistas à implantação de políticas de saúde pública, considerando as orientações publicadas na Atualização da Diretriz Brasileira de Dislipidemias e Prevenção da Aterosclerose de 2017, sem considerar outros fatores relacionados, como histórico de hiperlipemias de origem genética. A continuidade deste estudo se dará mediante avaliação de aspectos ligados ao perfil lipídico e análise do risco de doenças cardiovasculares na infância e na juventude por meio de biomarcadores como proteína $\mathrm{C}$ reativa, apolipoproteínas B e A-I, fibrinogênio, TNF e IL-6.

O perfil de lipoproteínas na infância e adolescência não sofre influência do fator regional, pois a prevalência de dislipidemias é semelhante a outras regiões do Brasil, ficando entre 22 e 35\%.

O percentual de dislipidemias na infância registrado em diversas regiões do país aponta a necessidade de implantação de políticas públicas para a prevenção de dislipidemia juvenil com o objetivo de minimizar agravos relacionados a doenças cardiovasculares na vida adulta da população.

\section{REFERÊNCIAS}

1. Faludi AA et al. Atualização da Diretriz Brasileira de Dislipidemias e Prevenção da Aterosclerose - 2017. Arq Bras Cardiol. 2017;109(2 Suppl. 1), 1-76.

2. Giuliano IC, Coutinho MS, Freitas SF, Pires MM, Zunino JN, Ribeiro RQ Lípides séricos em crianças e adolescentes da rede escolar de Florianópolis - estudo Floripa Saudável 2040. Arq Bras Cardiol. 2005;85(2):85-91. http:// dx.doi.org/10.1590/S0066-782X2005001500003. PMid:16113845.

3. Sociedade Brasileira de Cardiologia. I Diretriz de Prevenção da Aterosclerose na Infância e na Adolescência. Arq Bras Cardiol. 2005;85(Suppl. 6):1-36. http://dx.doi.org/10.1590/S0066-782X2005002500001.

4. Uiterwaal CS, Witteman JC, de Bruijn AM, Hofman A, Grobbee DE Families and natural history of lipids in childhood: an 18-year followup study. Am J Epidemiol. 1997;145(9):777-85. http://dx.doi.org/10.1093/ oxfordjournals.aje.a009170. PMid:9143207.

5. Brotons C, Ribera A, Perich RM, Abrodos D, Magana P, Pablo S, et al. Worldwide distribution of blood lipids and lipoproteins in childhood and adolescence: a review study. Atherosclerosis. 1998;139(1):1-9. http://dx.doi. org/10.1016/S0021-9150(98)00056-2. PMid:9699886

6. Rover MRM, Kupek E, Delgado RCB, Souza LC. Perfil lipídico e sua relação com fatores de risco para a aterosclerose em crianças e adolescentes. Revista Brasileira de Análises Clínicas. 2010;42(3):191-5.

7. Holman RL, McGill HC Jr, Strong JP, Geer JC. The natural history of atherosclerosis. Am J Pathol. 1958;34(2):209-35. PMid:13520905.

8. Stary HC. The sequence of cell and matrix changes in atherosclerotic lesions of coronary arteries in the first forty years of life. Eur Heart J. 1990(11 Suppl E):3-19. http://dx.doi.org/10.1093/eurheartj/11.suppl_E.3.

9. Kannel WB, Wilson PWF. An update on coronary risk factors. Med Clin North Am. 1995;79(5):951-71. http://dx.doi.org/10.1016/S0025-7125(16)30016-5. PMid:7674694.

10. Tracy RE, Newman WP 3rd, Wattigney WA, Berenson GS. Risk factors and atherosclerosis in youth autopsy findings of the Bogalusa Heart Study. Am
J Med Sci. 1995;310(Suppl 1):37-41. http://dx.doi.org/10.1097/00000441199512000-00007. PMid:7503122.

11. Castelli WP, Garrison RJ, Wilson PWF, Abbott RD, Kalousdian S, Kannel WB. Incidence of coronary heart disease and lipoprotein cholesterol levels. The Framingham Study. JAMA. 1986;256(20):2835-8. http://dx.doi. org/10.1001/jama.1986.03380200073024. PMid:3773200.

12. Menotti A, Lanti M, Puddu PE, Kromhout D. Coronary heart disease incidence in northern and southern European population: a reanalysis of seven countries study for a European coronary risk chart. Heart. 2000;84(3):238-44. http://dx.doi.org/10.1136/heart.84.3.238. PMid:10956281.

13. Chen W, Berenson GS. Metabolic syndrome: definition and prevalence in children. J Pediatr (Rio J). 2007;83(1):1-2. http://dx.doi.org/10.2223/ JPED.1584. PMid:17279287.

14. Knuiman JT, Hermus RJ, Hautvast JG. Serum total and high density lipoprotein (HDL) cholesterol concentrations in rural and urban boys from 16 countries. Atherosclerosis. 1980;36(4):529-37. http://dx.doi. org/10.1016/0021-9150(80)90245-2. PMid:7417370.

15. Schulpis K, Karikas GA. Serum cholesterol and triglyceride distribution in 7767 school-aged Greek children. Pediatrics. 1998;101(5):861-4. http:// dx.doi.org/10.1542/peds.101.5.861. PMid:9565415.

16. Lauer RM, Lee J, Clarke WR. Factors affecting the relationship between childhood and adult cholesterol levels: the Muscatine Study. Pediatrics. 1988;82(3):309-18. PMid:3405659.

17. Boreham C, Twisk J, Neville C, Savage M, Murray L, Gallagher A. Associations between physical fitness and activity patterns during adolescence and cardiovascular risk factors in young adulthood: the Northern Ireland Young Hearts Project. Int J Sports Med. 2002;23(S1):S22-6. http://dx.doi. org/10.1055/s-2002-28457. PMid:12012258.

18. Freedman DS, Khan LK, Dietz WH, Srinivasan SR, Berenson GS. Relationship of childhood obesity to coronary heart disease risk factors in adulthood: the Bogalusa Heart Study. Pediatrics. 2001;108(3):712-8. http://dx.doi. org/10.1542/peds.108.3.712. PMid:11533341. 
19. Instituto Brasileiro de Geografia e Estatistica. Censo demográfico 2010. Características da população e dos domicílios: resultados do universo. Rio de Janeiro: IBGE; 2011. CD-ROM.

20. Gerber ZR, Zielinsky P. Fatores de risco de aterosclerose na infância. Um estudo epidemiológico. Arq Bras Cardiol. 1997;69(4):231-6. http://dx.doi. org/10.1590/S0066-782X1997001000002. PMid:9595714.

21. Caramelli B, Giuliano ICB. Dislipidemia em crianças e adolescentes. Rev Soc Cardiol Estado de São Paulo. 2005;6:518-23.

22. Dennison BA, Kikuchi DA, Srinivasan SB, Webber LS, Berenson GS. Parental history of cardiovascular disease as indication for screening for lipoproteína abnormalities in children. J Pediatr. 1989;115(2):186-94. http:// dx.doi.org/10.1016/S0022-3476(89)80063-0. PMid:2754548.

23. Bezerra AC, Sampaio HAC, Melo MLP, Maia CSC, Almeida PC. Associaçao entre dislipidemia e excesso de peso de crianças e adolescentes atendidos em uma unidade de saúde. Rev Baiana de Saúde Pública. 2011;35(2):348-62.

24. Faria EC, Dalpino FB, Takata R. Lípides e lipoproteínas séricos em crianças e adolescentes ambulatoriais de um hospital universitário públlico. Rev Paul Pediatr. 2008;26(1):54-8. http://dx.doi.org/10.1590/S0103-05822008000100009.
25. Siqueira AFA, Abdalla DSP, Ferreira SRG. LDL: da síndrome metabólica à instabilização da placa aterosclerótica. Arq Bras Endocrinol Metabol. 2006;50(2):334-43. http://dx.doi.org/10.1590/S0004-27302006000200020. PMid:16767300.

26. Lima SCVC, Arrais RF, Almeida MG, Souza ZM, Pedrosa LFC. Perfi lipídico e peroxidação de lipídeos no plasma e crianças e adolescentes com sobrepeso e obesidade. J Pediatr (Rio J). 2004;80(1):23-8. http://dx.doi. org/10.2223/1129. PMid:14978545.

27. Ribas SA, Silva LCS. Dislipidemia em escolares na rede privada de Belém. Arq Bras Cardiol. 2009;92(6). http://dx.doi.org/10.1590/S0066782X2009000600006. PMid:19629307.

28. Pituelli Suárez N, Corbera Prin M, Lioi Luciani S, Turco Pilottó M, D’arrigo Dri M, Rosillo Politti I. Prevalencia de factores de riesgo: obesidade y perfil lipídico. An Pediatr (Barc). 2008;68(3):257-63. http://dx.doi. org/10.1157/13116706. PMid:18358137.

Recebido em: Mar. 20, 2018 Aprovado em: Mar. 27, 2019 